\title{
FACTORES DE RISCO: A MULTIPLICIDADE DAS VARIÁVEIS CONTEXTUAIS NO DESENVOLVIMENTO DAS CRIANÇAS
}

\author{
Joana Cadima ${ }^{1}$ \\ Carla Peixoto ${ }^{2}$ \\ Teresa Leal ${ }^{3}$
}

Resumo: O presente estudo pretende analisar os efeitos de múltiplos factores de risco do contexto familiar no desenvolvimento cognitivo e social de crianças em idade pré-escolar. Vários estudos têm demonstrado que as competências das crianças estão associadas a variáveis familiares permanecendo, no entanto, o debate sobre se as variáveis mais influentes correspondem a características particulares das famílias ou se o desenvolvimento das crianças depende, antes, da conjugação de diferentes factores. No âmbito do projecto Contextos e Transição foram avaliadas competências de literacia, numeracia e sociais de 95 crianças de 5 anos e recolhidas informações acerca de indicadores de risco nas respectivas famílias. As análises confirmaram que, à medida que aumenta o número de factores de risco, os resultados cognitivos das crianças tendem a ser mais baixos, o que apoia a visão de que o poder de cada factor depende da sua acumulação, sendo o desenvolvimento influenciado pelas inter-relações entre os factores considerados.

Palavras-chave: factores de risco, literacia, numeracia, competências sociais

Risk factors: The multiplicity of family variables in child development (Abstract): The present study intends to analyze the effects of family multiple risk factors on preschool children's cognitive and social development. Several studies have shown that children's skills are associated with family characteristics, despite the controversy about whether the most influential variables are family specific characteristics or rather that developmental outcomes lie on the combination of different factors, no single factor having sufficient explanatory power per se. The Project Contexts and Transition (Contextos e Transição) selected children from 60 preschools of the city of Porto. The literacy, numeracy and social skills of 95 five-year-old children were assessed. Family risk indicators were also collected. The

\footnotetext{
${ }^{1}$ Bolseira de Doutoramento da Fundação para a Ciência e Tecnologia (SFRH/BD/ 28870/2006),jcadima@fpce.up.pt

2 Bolseira de Doutoramento da Fundação para a Ciência e Tecnologia (SFRH/BD/ 30764/2006)

${ }^{3}$ Faculdade de Psicologia e de Ciências da Educação da Universidade do Porto 
analyses confirmed that as the number of risk factors increased, children's cognitive performance decreased. The results also showed that the power of a single factor or set of factors lies on their accumulation in children's lives.

Key-words: risk factors, literacy, numeracy, social skills

\section{Introdução}

Tem sido demonstrado que as crianças expostas a condições sócio-culturais adversas têm uma maior probabilidade de não adquirir as competências necessárias para o posterior sucesso educativo (Burchinal, Roberts, Hooper, \& Zeisel, 2000; Gutman, Sameroff, \& Cole, 2003). Essas condições estão associadas, nomeadamente, à pobreza e a baixos níveis de escolaridade da mãe, havendo, contudo, uma ênfase crescente na importância de considerar uma multiplicidade de factores quando analisamos o impacto do risco no desenvolvimento das crianças (Gutman, Sameroff, \& Cole, 2003; Sameroff \& Fiese, 2000; Sameroff, Seifer, Baldwin, \& Baldwin, 1993).

O risco sócio-cultural é conceptualizado como a ausência de oportunidades de desenvolvimento - ou a presença de ameaças ambientais - devido ao empobrecimento das interacções com os seus contextos e à ausência de experiências adequadas às necessidades e capacidades das crianças (Garbarino \& Ganzel, 2000).

Os factores de risco que têm sido identificados, por estarem associados a baixos resultados desenvolvimentais, são consistentes entre os vários estudos (Gutman, Sameroff, \& Eccles, 2002). Um desses factores, a desvantagem económica, é visto como um dos que mais comprometem o desenvolvimento das crianças, ao estar relacionado com outros factores familiares (Ackerman, Izard, Schoff, Youngstrom, \& Kogos, 1999; Lerner, 1997; Sameroff et al., 1993).

A investigação sugere também que as crianças cujas mães têm um nível de escolaridade baixo obtêm em média resultados mais baixos em testes estandardizados do que os seus pares e tendem a não concluir os estudos (Gutman et al., 2002).

O desemprego tende a diminuir os recursos e a precipitar problemas a nível da saúde mental e do bem-estar (Garbarino \& Ganzel, 2000), o que, se estiver correlacionado com o baixo nível académico a longo prazo, reforça ainda mais as limitações económicas.

Outros factores incluem a monoparentalidade, agregados familiares numerosos e acontecimentos de vida negativos, tendo estes um impacto negativo no desenvolvimento das crianças (Burchinal et al., 2000; Gutman et al., 2002). 
Contudo, como salientam Pianta e Walsh (1996), a situação de risco corresponde a uma probabilidade não sendo, por isso, um conceito estático, o que indica que o resultado que merece a nossa preocupação, nomeadamente o insucesso académico, ainda não ocorreu. Deste modo, salienta-se a importância da prevenção e da identificação em idade precoce das crianças em risco sócio-cultural, de modo a impedir a concretização da situação de risco.

Em termos de análise do impacto dos factores de risco no desenvolvimento, permanecem diferentes abordagens e modelos do risco sócio-cultural, apesar de se reconhecer a importância de diferentes variáveis familiares no desenvolvimento das crianças (Ackerman et al., 1999; Burchinal et al., 2000; Hooper, Burchinal, Roberts, Zeisel, \& Neebe, 1998).

$\mathrm{O}$ modelo aditivo examina as relações únicas entre múltiplos aspectos do ambiente adverso e problemas no desenvolvimento (Ackerman et al., 1999). Este modelo apresenta, no entanto, a desvantagem de não considerar que, usualmente, os factores não ocorrem isoladamente, mas tendem antes a agrupar-se nos mesmos indivíduos (Gutman et al., 2002; Sameroff \& Fiese, 2000).

O modelo cumulativo, proposto por Sameroff e colaboradores (Gutman et al., 2003; Gutman et al., 2002; Sameroff \& Fiese, 2000; Sameroff et al., 1993), tem em conta esta co-ocorrência dos factores de risco, analisando a conjugação de diferentes factores, em vez de cada factor individual. Desta forma, neste modelo, o poder de cada factor de risco depende da acumulação dos diferentes factores.

Vários estudos têm evidenciado a pertinência deste modelo, mostrando que o número de factores de risco é mais relevante para a determinação do resultado do desenvolvimento do que qualquer factor de risco tomado isoladamente (Burchinal et al., 2000; Garbarino \& Ganzel, 2000; Gutman et al., 2003; Gutman et al., 2002; Hooper et al., 1998; Sameroff \& Fiese, 2000; Sameroff et al., 1993). Alguns estudos demonstraram ainda que os mesmos resultados eram obtidos com diferentes combinações de factores de risco, o que aponta para a falibilidade de intervenções universais e a necessidade de análises e intervenções únicas para cada criança e cada família (Sameroff \& Fiese, 2000; Sameroff et al., 1993).

Consequentemente, será importante compreender como os diversos factores podem afectar o desenvolvimento da criança, de modo a identificar precocemente as crianças em risco (Hooper et al., 1998) e facilitar o acesso a intervenções realmente eficazes no que respeita a criar oportunidades ou mobilizar recursos para uma determinada criança, família ou comunidade.

Dado que a literatura tem evidenciado a relevância de adoptar uma perspectiva abrangente do desenvolvimento, que considere a multiplicidade de factores associados à vida das crianças e respectivas famílias, o presente 
estudo pretende analisar os efeitos de múltiplos factores de risco do contexto familiar no desenvolvimento cognitivo e social de crianças em idade pré-escolar.

Mais especificamente, pretende-se analisar a utilidade do modelo cumulativo ou de múltiplo risco, proposto por Sameroff e colaboradores (Gutman et al., 2003; Sameroff \& Fiese, 2000) e verificar se a acumulação dos diferentes factores de risco determina resultados desenvolvimentais mais baixos, contribuindo para colocar a criança em situação de risco.

\section{Metodologia}

\section{Participantes}

O trabalho agora apresentado desenvolveu-se no âmbito de um projecto de investigação mais global denominado de Contextos e Transição: Competências de literacia e de numeracia em crianças dos 4 aos 7 anos, desenvolvido pelo Centro de Psicologia da Universidade do Porto (Subgrupo "Desenvolvimento: Contextos Familiares e Educativos"). Este projecto de investigação teve como objectivo geral estudar longitudinalmente competências de literacia, numeracia e sociais no final do pré-escolar e o seu valor preditivo no sucesso escolar e envolveu um total de 215 crianças a frequentar a educação pré-escolar.

O processo de selecção da amostra foi multi-etápico, tendo sido seleccionadas, em primeiro lugar, de forma aleatória, 60 salas de jardim-de-infância do Grande Porto e, posteriormente, cerca de 4 crianças por sala.

O presente estudo utiliza uma sub-amostra do projecto de investigação $(N=95)$, tendo sido seleccionadas as crianças que estavam a frequentar o último ano da educação pré-escolar (nascidas em 2000) e sobre as quais dispúnhamos de toda a informação ao nível dos vários instrumentos utilizados. A idade cronológica das crianças participantes variou entre 65 e 78 meses $(M=71 ; D P=3.4)$. Relativamente ao género, 52 crianças são do sexo masculino $(54.7 \%)$ e 43 crianças são do sexo feminino (45.3\%).

\section{Instrumentos}

Foram utilizados diferentes instrumentos para avaliar as competências de literacia e numeracia e os comportamentos das crianças no jardim-de-infância. Foram ainda recolhidas informações acerca dos indicadores de risco.

Relativamente às competências de literacia, utilizou-se a adaptação portuguesa da versão espanhola Test de vocabulário en imagénes Peabody (PPVT; Dunn, 1986), usada no âmbito do Estudo Europeu sobre Educação e 
Cuidados de Crianças em Idade Pré-Escolar (ECCE Study Group, 1997), com o objectivo de avaliar o vocabulário receptivo das crianças. Nesta prova, são apresentados cartões com quatro imagens cada, e é pedido à criança para apontar para a imagem que corresponde à palavra dita pelo avaliador.

Para avaliação do conhecimento das crianças sobre as convenções da escrita, recorreu-se à prova Conceitos sobre a Escrita - Segue-me Lua (versão para investigação, Alves e Aguiar, 2003), adaptação portuguesa de Follow me, Moon (Clay, 2000). Este instrumento avalia o reconhecimento das convenções e das características da linguagem escrita (tais como: direcção da leitura, correspondência entre palavras escritas e faladas, sinais de pontuação, conceito de letra, conceito de palavra) no decorrer de uma tarefa de leitura de um livro elaborado para o efeito.

A consciência fonológica foi avaliada através de uma tarefa experimental desenvolvida por Sucena e Castro (2001). São apresentados pares de palavras em voz alta e é pedido à criança que diga se o primeiro segmento nas duas palavras é igual ou não (consciência implícita), e posteriormente, que identifique o segmento que é igual (consciência explícita).

Para avaliar a identificação de letras, recorremos à tarefa de leitura de letras das Provas de Avaliação da Leitura - Nível Principiante (Castro, Cary, \& Gomes, 1998). As 21 letras maiúsculas do alfabeto português foram apresentadas duas vezes às crianças, em listas paralelas e ordens pseudo-aleatórias diferentes, sendo pedido à criança para dizer o nome ou o som das letras.

Quanto às competências de numeracia, foi utilizada a prova de aritmética da WPPSI-R, uma prova de Leitura de Números e uma prova de Contagem Abstracta. O teste de aritmética da WPPSI-R é constituído por três partes com diferentes tipos de tarefas: a) a criança deve apontar, de entre um conjunto de objectos apresentados visualmente, para aquele que possui a característica quantitativa enunciada verbalmente (maior, mais alto); b) avalia conhecimentos numéricos através da contagem e da manipulação de objectos; e c) resolução de problemas de aritmética apresentados oralmente. A prova de leitura de números consistiu em apresentar à criança uma série de 20 cartões com números escritos do 0 até 100 , para que ela indicasse de que número se tratava. Na prova de contagem, pediu-se à criança para contar até onde soubesse, obtendo-se a dimensão da sequência numérica. Admitia-se um erro e pedia-se à criança para parar de contar no número 111 .

Para avaliar os comportamentos das crianças utilizou-se o Inventário de Comportamentos na Sala (ICS), adaptação portuguesa do Classroom Behaviour Inventory (CBI; Schaefer, Edgerton, \& Aaronson, 1978). A versão pré-escolar é constituída por 60 itens, correspondendo cada um a uma afirmação sobre um determinado comportamento da criança (ex. Está quase sempre bem disposto/a e alegre). O educador de infância de cada criança 
avalia, através de uma escala de tipo Likert de 5 pontos $(1=$ Nunca assim; 5 = Sempre assim), o grau em que se observa na criança cada um dos comportamentos descritos. Foram identificados, no estudo da versão portuguesa (Abreu-Lima \& Cadima, 2006), quatro factores que correspondem às quatro subescalas usadas no presente estudo: Empenhamento, cujos itens exprimem o interesse pelas actividades da sala por parte da criança, motivação, persistência e autonomia na realização de tarefas; Inteligência, que agrupa itens como compreender palavras dificeis e possuir bons conhecimentos para a idade; Sociabilidade, onde estão agrupados itens que descrevem a criança na sua relação com os outros; Respeito pelos Outros, que inclui itens que se referem a comportamentos que mostram cuidado e atenção para com o outro.

Por fim, no sentido de recolher informação acerca dos indicadores de risco das crianças avaliadas foi elaborado o Questionário de Índice Multi-Risco. Este questionário incluiu informação acerca da família da criança identificada pela literatura como relevante para a situação de risco (Burchinal et al., 2000; Gutman, Sameroff, \& Cole, 2003; Sameroff \& Fiese, 2000) e que os educadores de infância conheciam ou poderiam facilmente obter: composição do agregado familiar, nível de escolaridade e ocupação profissional de ambos os pais ou dos principais prestadores de cuidados, rendimento económico do agregado familiar, língua falada em casa, nacionalidade e etnia da criança, uma listagem de 16 acontecimentos de vida negativos (ex. separação/divórcio dos pais, alcoolismo, abuso de drogas, internamento hospitalar) e ainda uma escala subjectiva de 3 pontos, em que se pedia ao educador de infância para avaliar a relação entre o principal prestador de cuidados e a criança, tendo como base a verificação de descuido/negligência na prestação de cuidados básicos (alimentação, higiene, vestuário, rotinas e sono). Alguma desta informação não foi posteriormente utilizada, nomeadamente, a nacionalidade e a etnia, uma vez que existia homogeneidade na amostra.

\section{Procedimento}

Depois de obtidas as devidas autorizações para a realização deste estudo, deu-se início à recolha de dados, a qual decorreu entre os meses de Maio e Julho de 2006. Os instrumentos foram administrados individualmente numa sala à parte dos respectivos jardins-de-infância. Ambos os questionários foram preenchidos pelas educadoras das crianças observadas.

\section{Factores de risco}

Foi calculado o índice multi-risco a partir da soma de oito factores de risco (cf. abaixo). Cada factor foi convertido em variável dicotómica $(1=$ presença, $0=$ ausência). No caso das variáveis contínuas, Sameroff e colabo- 
radores (2002) sugerem que, quando não é possível definir categorias objectivas, o critério que deve presidir é estatístico, sendo um quinto da amostra $(20 \%)$ incluída na categoria de risco.

O número de factores de risco encontrado variou entre 0 e $7(M=$ $1.87 ; D P=1.69$ ). Verificou-se que $25 \%$ da amostra não tem qualquer factor de risco, $21 \%$ tem um factor de risco, $25 \%$ tem dois, $12 \%$ tem três, $10 \%$ tem quatro e $7 \%$ entre cinco e sete factores de risco.

Apresentamos de seguida cada um dos factores de risco analisados.

1. Ausência de 1 ou de 2 progenitores (12.6\%): Foram consideradas em risco as crianças de famílias monoparentais $(8.4 \%)$, ou que não viviam com ambos os progenitores (4.2\%).

2. Nivel de escolaridade da mãe ou do principal prestador de cuidados inferior a 6 anos (47.4\%): Um nível de escolaridade da mãe igual ao $2^{\circ}$ ciclo $(28.4 \%)$ ou inferior (analfabetas $=1.1 \%$ e $1^{\circ}$ ciclo $=17.9 \%$ ) foi identificado como factor de risco.

3. Ocupação profissional desqualificada do principal prestador de cuidados $(51.6 \%)$ : Foram consideradas factor de risco as ocupações profissionais como doméstica, desempregada ou reformada (29.5\%) e os trabalhadores não qualificados $(22.1 \%)$.

4. Família com subsídios da Segurança Social ou rendimentos inferiores ao salário mínimo (11.6\%): Incluímos na categoria de risco as crianças de famílias que dependem de subsídios da Segurança Social (7.4\%) ou com rendimentos inferiores ao salário mínimo (4.2\%).

5. Elevado número de crianças a viver em casa (12.6\%): As famílias com 3 ou mais crianças (menores de 18 anos) a viverem em casa foram consideradas em risco.

6. Eventos familiares negativos (34.7\%): Foram incluídas nesta categoria as famílias com um $(20,0 \%)$ ou mais eventos familiares negativos (dois $=6.3 \%$, três $=5.3 \%$, quatro $=2.1 \%$, seis $=1.1 \%$ ).

7. Verificação, por parte do educador de infância, de situações de descuido/negligência relativamente à prestação de cuidados (higiene, alimentação, vestuário) (7.4\%): Situações de descuido e negligência ocasional $(4.2 \%)$ e grave $(3.2 \%)$ relativamente à prestação de cuidados foram consideradas factor de risco.

8. Fraca assiduidade ao jardim-de-infância (9.5\%): Relativamente a este factor, incluímos as crianças que faltavam algumas vezes e chegavam tarde $(1.1 \%)$, as crianças que faltavam frequentemente e/ou chegavam tarde $(8.5 \%)$. 


\section{Resultados}

Correlações entre os factores de risco e os resultados desenvolvimentais das crianças

Foram analisadas inicialmente as associações existentes entre cada factor de risco, o índice multi-risco e os resultados das crianças a nível das diferentes competências de literacia, de numeracia e dos comportamentos exibidos na sala de jardim-de-infância. Análises preliminares permitiram verificar a existência de associações positivas estatisticamente significativas entre os resultados desenvolvimentais das crianças e a idade cronológica, através do cálculo de coeficientes de correlação momento produto de Pearson $(r>0,20$, excepto para a prova de aritmética da WPPSI-R e a Sociabilidade). Da mesma forma, no que se refere ao género das crianças, verificou-se existirem associações estatisticamente significativas com os resultados de numeracia e da sub-escala Respeito pelos Outros $(r>0,22)$. Tendo sido obtidos coeficientes de correlação estatisticamente significativos, decidimos determinar as associações entre os vários factores de risco e os resultados obtidos a nível das diferentes competências, mantendo os efeitos da idade cronológica e do género constantes. São apresentados no Quadro 1 os coeficientes de correlação parcial obtidos, assim como as médias e os desvios-padrão das provas aplicadas às crianças.

Apesar das associações entre os factores de risco e as variáveis desenvolvimentais variarem na sua intensidade e no seu nível de significância estatística, cada factor de risco apresenta associações negativas com, pelo menos, duas das variáveis desenvolvimentais, conforme se apresenta no Quadro 1. É ainda de referir que os factores de risco que se correlacionam negativamente com um maior número de resultados das crianças são o Factor de Risco 4, que diz respeito a baixos rendimentos económicos, sendo sete dos onze coeficientes de correlação estatisticamente significativos e o Factor de Risco 2, correspondente a um nível de escolaridade da mãe inferior a seis anos, que apresenta associações negativas com cinco das onze variáveis desenvolvimentais.

Relativamente ao índice multi-risco, é de salientar que os coeficientes de correlação parcial obtidos entre as várias competências cognitivas e o índice multi-risco são mais fortes do que entre essas mesmas competências e qualquer um dos factores individuais (cf. Quadro 1). Verifica-se a existência de associações negativas com os diferentes resultados desenvolvimentais, excepto com os resultados obtidos a nível dos comportamentos sociais (Sociabilidade e Respeito pelos Outros), em que não existem associações estatisticamente significativas. Relativamente ao Empenhamento, o coefi- 
ciente de correlação é tendencialmente significativo. Estas análises sugerem que a acumulação de factores de risco terá essencialmente implicações a nível cognitivo, mais do que no âmbito social.

Quadro 1. Médias, Desvio-Padrão e Coeficientes das correlações parciais entre as competências de literacia emergente, de numeracia e os comportamentos das crianças $(N=95)$, com o controlo dos efeitos da idade cronológica e do género

\begin{tabular}{|c|c|c|c|c|c|c|c|c|c|c|c|}
\hline & \multicolumn{4}{|c|}{$\begin{array}{c}\text { Competências de } \\
\text { Literacia } \\
\end{array}$} & \multicolumn{3}{|c|}{$\begin{array}{l}\text { Competências } \\
\text { de numeracia }\end{array}$} & \multicolumn{4}{|c|}{$\begin{array}{c}\text { Comportamentos } \\
\text { na sala } \\
\end{array}$} \\
\hline & 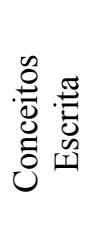 & 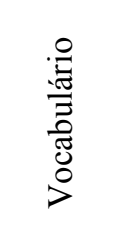 & 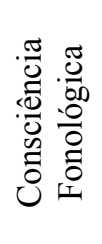 & 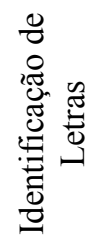 & $\begin{array}{l}\frac{x}{1} \\
\frac{n}{2} \\
\frac{1}{3}\end{array}$ & 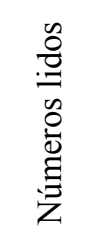 & 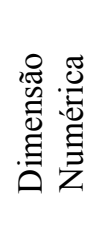 & 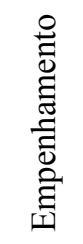 & 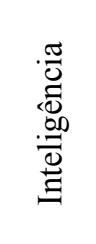 & $\begin{array}{l}\frac{0}{0} \\
\frac{\pi}{0} \\
:= \\
: \frac{\pi}{7} \\
0 \\
0 \\
0\end{array}$ & 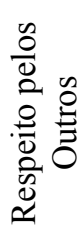 \\
\hline $\begin{array}{l}\text { Factor } \\
\text { Risco_1 }\end{array}$ & -.04 & $-.18^{\dagger}$ & $-.19^{\dagger}$ & $-.23 *$ & $-.19^{\dagger}$ & -.13 & $-.22 *$ & -.05 & -.09 & .06 & -.01 \\
\hline $\begin{array}{l}\text { Factor } \\
\text { Risco_2 }\end{array}$ & $-.18^{\dagger}$ & $-.24 *$ & $-.17^{\dagger}$ & $-.25^{*}$ & $-.20^{\dagger}$ & $-.27 * *$ & -.14 & $-.21^{*}$ & $-.23^{*}$ & -.03 & $-.19^{\dagger}$ \\
\hline $\begin{array}{l}\text { Factor } \\
\text { Risco_3 }\end{array}$ & -.08 & -.09 & -.14 & $-.25^{*}$ & -.14 & $-.25^{*}$ & -.16 & -.12 & -.17 & -.17 & -.08 \\
\hline $\begin{array}{l}\text { Factor } \\
\text { Risco_4 }\end{array}$ & -.13 & $-.34 * *$ & $-.29 * *$ & $-.28 * *$ & $-.31 * *$ & $-.23 *$ & $-.24^{*}$ & -.08 & $-.18^{\dagger}$ & $-.23 *$ & -.04 \\
\hline $\begin{array}{l}\text { Factor } \\
\text { Risco_5 }\end{array}$ & -.03 & $-.31 * *$ & -.15 & -.09 & $-.23 *$ & $-.20^{\dagger}$ & -.14 & -.01 & -.11 & -.10 & -.02 \\
\hline $\begin{array}{l}\text { Factor } \\
\text { Risco_6 }\end{array}$ & $-.22 *$ & $-.25^{*}$ & -.11 & $-.22 *$ & $-.27 * *$ & -.15 & -.12 & -.02 & -.04 & .11 & $.18^{\dagger}$ \\
\hline $\begin{array}{l}\text { Factor } \\
\text { Risco_7 }\end{array}$ & $-.20^{\dagger}$ & $-.18^{\dagger}$ & -.13 & -.10 & $-.26^{*}$ & -.10 & $-.24 *$ & $-.18^{\dagger}$ & $-.22 *$ & $-.19^{\dagger}$ & -.07 \\
\hline $\begin{array}{l}\text { Factor } \\
\text { Risco_8 }\end{array}$ & -.11 & -.17 & -.13 & -.07 & $-.18^{\dagger}$ & -.14 & -.07 & $-.21 *$ & $-.24 *$ & -.17 & -.05 \\
\hline $\begin{array}{l}\text { Índice de } \\
\text { Risco }\end{array}$ & $-.21^{*}$ & $-.39 * * *$ & $-.29 * *$ & $-.35 * *$ & $-.39 * * *$ & $-.34 * *$ & $-.29 * *$ & $-.20^{\dagger}$ & $-.28 * *$ & -.14 & -.01 \\
\hline$M$ & 7.72 & 44.24 & 2.97 & 7.43 & 7.86 & 38.00 & 40.49 & 3.80 & 3.78 & 4.11 & 3.63 \\
\hline$D P$ & 3.24 & 15.25 & 1.84 & 7.27 & 2.39 & 24.02 & 24.02 & .69 & .75 & .61 & .74 \\
\hline
\end{tabular}

Notas: ${ }^{\dagger} p<0,10 ; * p<0,05 ; * * p<0,01 ; * * * p<0,001$ 


\section{Análises de regressão múltipla hierárquica}

Com o objectivo de testar os efeitos do índice multi-risco nos resultados desenvolvimentais das crianças, recorremos ao modelo de regressão múltipla hierárquica, cujos resultados, para as competências de literacia, de numeracia e os comportamentos na sala são apresentados, respectivamente, nos Quadros 2, 3 e 4. Para cada resultado das crianças, as análises de regressão incluíram dois grupos de variáveis: o primeiro bloco incluiu a idade cronológica e o género das crianças, sendo incluído no segundo bloco o índice multi-risco. Foi deste modo possível determinar a percentagem de variação nos resultados explicada pelo índice multi-risco, depois de terem sido controlados os efeitos da idade e do género.

Competências de literacia: Como mostra o Quadro 2, a mudança em $R^{2}$ foi estatisticamente diferente de zero no final do $2^{\circ}$ bloco ao nível das quatro competências de literacia, o que indica que o índice multi-risco efectua uma contribuição estatisticamente significativa para todas as variáveis de literacia emergente: nos conceitos sobre a escrita, o índice multi-risco explica $4 \%(p<0,05)$ da variação nos resultados, no vocabulário receptivo, $13 \%$ $(p<0,001)$, na consciência fonológica, $8 \%(p<0,01)$ e na identificação de letras $11 \%(p<0,01)$. A interpretação dos valores $\beta$ permitiu confirmar que o índice multi-risco é um preditor significativo dos conceitos sobre a escrita, $\beta=-0,20, p<0,05$, do vocabulário receptivo, $\beta=-0,36, p<0,001$, da consciência fonológica, $\beta=-0,28, p<0,01$ e da identificação das letras, $\beta$ $=-0,33, p<0,01$ (cf. Quadro 2). Assim, parece que quanto maior o número de riscos presentes na vida das crianças, mais baixos são os resultados obtidos a nível das diferentes provas de literacia.

Competências de numeracia: Os resultados das regressões múltiplas hierárquicas para as variáveis de numeracia revelaram que o índice multi-risco efectua uma contribuição significativa: $15 \%(p<0,001)$ da variação nos resultados da prova de aritmética da WPPSI-R é explicada com a introdução do índice multi-risco no modelo de regressão, $10 \%(p<0,01)$ nos resultados da leitura de números e 7\% $(p<0,01)$ nos resultados da dimensão numérica (cf. Quadro 3). As análises dos coeficientes estandardizados indicam que o índice multi-risco tem um poder explicativo estatisticamente significativo na prova da WPPSI-R, $\beta=-0,38, p<0,001$, na leitura de números, $\beta=-0,32, p<0,01$ e na dimensão da sequência numérica, $\beta=-0,27$, $p<0,01$. Neste sentido, à medida que as crianças estão expostas a um maior número de factores de risco, os resultados nas provas de numeracia decrescem. 
Quadro 2. Sumário da análise de regressão múltipla hierárquica para predição dos resultados de competências de literacia

\begin{tabular}{|c|c|c|c|c|c|c|c|}
\hline \multirow[t]{2}{*}{ Variáveis } & \multicolumn{3}{|c|}{ Conceitos Escrita } & \multicolumn{4}{|c|}{ Vocabulário Receptivo } \\
\hline & $B$ & $S E$ & $\beta$ & $B$ & $S E$ & $\beta$ & \\
\hline Passo 1 & & & $R^{2}=.09 *$ & & & & $R^{2}=.10 * *$ \\
\hline $\begin{array}{l}\text { Idade da } \\
\text { criança }\end{array}$ & .27 & .09 & $.28 * *$ & 1.34 & .44 & $.30 * *$ & \\
\hline $\begin{array}{l}\text { Género da } \\
\text { criança }\end{array}$ & -.49 & .65 & -.08 & -2.10 & 3.1 & -.07 & \\
\hline Passo 2 & & & $\Delta R^{2}=.04 *$ & & & & $\Delta R^{2}=.13 * * *$ \\
\hline $\begin{array}{l}\text { Idade da } \\
\text { criança }\end{array}$ & .28 & .09 & $.29 * *$ & 1.41 & .41 & $.32 * *$ & \\
\hline $\begin{array}{l}\text { Género da } \\
\text { criança }\end{array}$ & -.51 & .64 & -.08 & -2.23 & 2.85 & -.07 & \\
\hline $\begin{array}{l}\text { Índice de } \\
\text { Risco }\end{array}$ & -.38 & .19 & $-.20 *$ & -3.40 & .88 & $-.36 * * *$ & \\
\hline \multirow[t]{2}{*}{ Variáveis } & \multicolumn{3}{|c|}{ Consciência Fonológica } & \multicolumn{4}{|c|}{ Identificação de letras } \\
\hline & $B$ & $S E$ & $\beta$ & $B$ & $S E$ & $\beta$ & \\
\hline Passo 1 & & & $R^{2}=.05$ & & & & $R^{2}=.13^{* *}$ \\
\hline $\begin{array}{l}\text { Idade da } \\
\text { criança }\end{array}$ & .11 & .06 & $.21^{*}$ & .78 & .21 & $.37 * * *$ & \\
\hline $\begin{array}{l}\text { Género da } \\
\text { criança }\end{array}$ & -.17 & .38 & -.05 & .28 & 1.4 & .02 & \\
\hline Passo 2 & & & $\Delta R^{2}=.08^{* *}$ & & & & $\Delta R^{2}=.11^{* *}$ \\
\hline $\begin{array}{l}\text { Idade da } \\
\text { criança }\end{array}$ & .12 & .06 & $.22^{*}$ & .81 & .19 & $.38 * * *$ & \\
\hline $\begin{array}{l}\text { Género da } \\
\text { criança }\end{array}$ & -.19 & .37 & -.05 & .20 & 1.3 & .01 & \\
\hline $\begin{array}{l}\text { Índice de } \\
\text { Risco }\end{array}$ & -.30 & .11 & $\begin{array}{l}-.28 \\
* * \\
\end{array}$ & -1.4 & .39 & $-.33 * *$ & \\
\hline
\end{tabular}

Notas: ${ }^{\dagger} p<0,10 ; * p<0,05, * * p<0,01 ; * * * p<0,001 ;$ (género da criança: $0=$ masculino)

Comportamentos na sala: A partir da análise do Quadro 4, que apresenta os modelos de regressão para as subescalas do comportamento das crianças na sala de jardim de infância, verifica-se que a contribuição do índice multi-risco é significativa apenas na subescala Inteligência (mudança em $\left.R^{2}=7 \%, p<0,01\right)$, e tendencialmente significativa na subescala Empenhamento, explicando $3 \%(p<0,10)$ da variação nos resultados nesta subescala. No mesmo sentido, o índice multi-risco é um preditor significativo na subes- 
cala Inteligência $(\beta=-0,27, p<0,01)$ e tendencialmente significativo na subescala Empenhamento $(\beta=-0,19, p<0,10)$, mostrando uma certa tendência para os resultados do Empenhamento decrescerem à medida que aumenta o número de riscos, sendo este efeito mais notório ao nível da subescala Inteligência. Relativamente à Sociabilidade e Respeito pelos Outros, a introdução do índice multi-risco nos modelos de regressão não melhorou os seus valores preditivos, verificando-se que esta variável não efectua uma contribuição estatisticamente significativa nestes resultados.

Quadro 3. Sumário da análise de regressão múltipla hierárquica para predição dos resultados de competências de numeracia

\begin{tabular}{|c|c|c|c|c|c|c|c|c|c|}
\hline \multirow[t]{2}{*}{ Variáveis } & \multicolumn{3}{|c|}{ WPPSI-R } & \multicolumn{3}{|c|}{ Leitura de números } & \multicolumn{3}{|c|}{$\begin{array}{l}\text { Dimensão da } \\
\text { sequência numérica }\end{array}$} \\
\hline & $B$ & $S E$ & $\beta$ & $\bar{B}$ & $S E$ & $\beta$ & $B$ & $S E$ & $\beta$ \\
\hline Passo 1 & & & $R^{2}=.03$ & & & $R^{2}=.13 * *$ & & & $R^{2}=.15^{* *}$ \\
\hline $\begin{array}{l}\text { Idade da } \\
\text { criança }\end{array}$ & .01 & .07 & .01 & 1.81 & .71 & $.25^{*}$ & 2.19 & .68 & $.31 * *$ \\
\hline $\begin{array}{l}\text { Género da } \\
\text { criança }\end{array}$ & -.81 & .49 & -.17 & -11.65 & 4.87 & $-.23^{*}$ & -8.80 & 4.66 & $-.18^{\dagger}$ \\
\hline Passo 2 & & & $\Delta R^{2}=.15 * * *$ & & & $\Delta R^{2}=.10^{* *}$ & & & $\Delta R^{2}=.07 * *$ \\
\hline $\begin{array}{l}\text { Idade da } \\
\text { criança }\end{array}$ & .02 & .07 & .03 & 1.88 & .67 & $.26^{* *}$ & 2.26 & .65 & $.32 * *$ \\
\hline $\begin{array}{l}\text { Género da } \\
\text { criança }\end{array}$ & -.83 & .46 & $-.17^{\dagger}$ & -11.88 & 4.60 & $-.24 *$ & -8.99 & 4.49 & $-.19 *$ \\
\hline $\begin{array}{l}\text { Índice de } \\
\text { Risco }\end{array}$ & -.54 & .13 & $-.38 * * *$ & -4.68 & 1.35 & $-.32 * *$ & -3.82 & 1.32 & $-.27 * *$ \\
\hline
\end{tabular}


Quadro 4. Sumário da análise de regressão múltipla hierárquica para predição dos resultados dos comportamentos na sala

\begin{tabular}{|c|c|c|c|c|c|c|c|c|}
\hline \multirow[t]{2}{*}{ Variáveis } & \multicolumn{3}{|c|}{ Empenhamento } & \multicolumn{5}{|c|}{ Inteligência } \\
\hline & $B$ & $S E$ & $\beta$ & & $B$ & $S E$ & $\beta$ & \\
\hline Passo 1 & & & & $R^{2}=.10^{* *}$ & & & & $R^{2}=.10^{* *}$ \\
\hline $\begin{array}{l}\text { Idade da } \\
\text { criança }\end{array}$ & .06 & .02 & $.30 * *$ & & .06 & .02 & $.31 * *$ & \\
\hline $\begin{array}{l}\text { Género da } \\
\text { criança }\end{array}$ & .21 & .14 & .15 & & .12 & .15 & .08 & \\
\hline Passo 2 & & & & $\Delta R^{2}=.03^{\dagger}$ & & & & $\Delta R^{2}=.07 * *$ \\
\hline $\begin{array}{l}\text { Idade da } \\
\text { criança }\end{array}$ & .06 & .02 & $.31 * *$ & & .07 & .02 & $.32 * *$ & \\
\hline $\begin{array}{l}\text { Género da } \\
\text { criança }\end{array}$ & .20 & .14 & .15 & & .12 & .14 & .07 & \\
\hline $\begin{array}{l}\text { Índice de } \\
\text { Risco }\end{array}$ & -.07 & .04 & $-.19^{\dagger}$ & & -.12 & .04 & $-.27 * *$ & \\
\hline \multirow[t]{2}{*}{ Variáveis } & \multicolumn{3}{|c|}{ Sociabilidade } & & \multicolumn{4}{|c|}{ Respeito pelos Outros } \\
\hline & $B$ & $S E$ & $\beta$ & & $B$ & $S E$ & $\beta$ & \\
\hline Passo 1 & & & & $R^{2}=.02$ & & & & $R^{2}=.11 * *$ \\
\hline $\begin{array}{l}\text { Idade da } \\
\text { criança }\end{array}$ & .02 & .02 & .13 & & .02 & .02 & .10 & \\
\hline $\begin{array}{l}\text { Género da } \\
\text { criança }\end{array}$ & -.02 & .13 & -.02 & & .49 & .15 & $.34 * *$ & \\
\hline Passo 2 & & & & $\Delta R^{2}=.02$ & & & & $\Delta R^{2}=.00$ \\
\hline $\begin{array}{l}\text { Idade da } \\
\text { criança }\end{array}$ & .02 & .02 & .14 & & .02 & .02 & .10 & \\
\hline $\begin{array}{l}\text { Género da } \\
\text { criança }\end{array}$ & -.02 & .13 & -.02 & & .49 & .15 & $.34 * *$ & \\
\hline $\begin{array}{l}\text { Índice de } \\
\text { Risco }\end{array}$ & -.05 & .04 & -.14 & & -.00 & .04 & -.01 & \\
\hline
\end{tabular}

Notas: ${ }^{\dagger} p<0,10 ; * p<0,05 ; * * p<0,01 ; * * * p<0,001$; (género da criança: $0=$ masculino)

\section{Discussão dos Resultados}

O presente estudo tinha por objectivo testar o poder preditivo da acumulação de factores de risco do contexto familiar no desenvolvimento cognitivo e social de crianças em idade pré-escolar.

As análises dos modelos de regressão permitiram verificar que o índice multi-risco tem um poder explicativo nos resultados das competências de 
literacia, de numeracia e ao nível da subescala Inteligência, sendo patente o impacto negativo da acumulação de factores de risco no desenvolvimento cognitivo das crianças. Estes resultados estão de acordo com outras investigações que indicam o impacto negativo da acumulação de factores de risco nos resultados das crianças (e.g., Gutman et al., 2003; Gutman et al., 2002; Sameroff \& Fiese, 2000).

Contudo, no presente estudo o índice multi-risco não assumiu um efeito estatisticamente significativo nas subescalas Sociabilidade e Respeito pelos Outros. A exibição destes comportamentos sociais parece ser independente de um maior número de factores de risco presentes na vida das crianças. Os nossos resultados, porém, não indicam que as características familiares avaliadas não sejam importantes para o desenvolvimento desses comportamentos. De facto, de acordo com as análises dos coeficientes de correlação entre cada factor de risco e os resultados das crianças, verifica-se uma associação negativa entre a Sociabilidade e um baixo rendimento económico (Factor de Risco 4) e uma associação tendencialmente negativa entre a Sociabilidade e a verificação de situações de descuido/negligência relativamente à prestação de cuidados (Factor de Risco 7). Existe ainda uma relação tendencialmente negativa entre um nível baixo de escolaridade da mãe (Factor de Risco 2) e o Respeito pelos Outros. Pode acontecer que estes aspectos da vida da criança assumam uma especial importância no desenvolvimento social, não se verificando um efeito cumulativo com os outros factores. É ainda surpreendente a associação tendencialmente positiva verificada entre os acontecimentos de vida negativos e o Respeito pelos Outros. Esta associação parece sugerir que, face a circunstâncias mais nefastas, as crianças podem desenvolver comportamentos que permitam lidar melhor com os outros. No entanto, colocamos algumas reservas na interpretação destes resultados e consideramos ser necessário investigar mais aprofundadamente, de modo a compreendermos melhor quais os factores influentes no desenvolvimento social.

Os resultados do presente estudo acentuam a importância de conceptualizar a situação de risco no quadro das influências recíprocas dos múltiplos factores presentes nos contextos de vida das crianças. Neste sentido, a acção de cada factor de risco é melhor compreendida na sua interacção com os outros factores de risco, estando o seu poder dependente da acumulação de factores na vida de uma determinada criança e da sua família. Consequentemente, os resultados apoiam a visão de que o desenvolvimento é produto das interacções dinâmicas e contínuas entre a criança e as experiências providenciadas pela família e pelo seu contexto social (Sameroff \& Fiese, 2000). Para promover o desenvolvimento, é necessário atender às características parentais, familiares e sociais que se influenciam mutuamente e que operam no contexto de vida de uma determinada criança, sendo importante 
que as medidas de intervenção sejam dirigidas a um problema particular para uma criança particular numa família e numa cultura particulares (Sameroff \& Fiese, 2000). Será por isso reducionista adoptar uma visão que considere ser possível implementar um único tipo de programa de intervenção, de modo a prevenir e a promover o desenvolvimento de todas as crianças. Dificilmente uma única acção conseguirá ir ao encontro de todas as exigências e necessidades das crianças (Garbarino \& Ganzel, 2000; Lerner, 1997).

Parece-nos, antes, essencial desenvolver uma variedade de acções junto, não só das crianças em situação de risco, como também das suas famílias, que propiciem, antes da actualização dos factores de risco (e, por isso, o mais precocemente possível), condições e oportunidades que assegurem e promovam o desenvolvimento futuro da criança.

Para promover o desenvolvimento destas crianças parece-nos, deste modo, imprescindível articular e optimizar as acções de profissionais de várias áreas, de modo a actuar a nível de diferentes factores, assim como articular diferentes serviços (como os de saúde, segurança social e educação) que, ao aliarem esforços e podendo actuar ao nível da família, poderão ter um papel crucial na mudança das trajectórias desenvolvimentais das crianças. Como refere Lerner (1997), é preciso integrar um pacote de serviços/programas que não devem estar apenas orientados para os intervenientes (crianças e família), mas também para as relações existentes e que influenciam o desenvolvimento das crianças - nomeadamente, as interacções entre a criança e os pais, a relação entre a escola e a família, etc. - e que poderão constituir-se como os recursos potenciais para a mudança.

No entanto, ao implicar que se conjugue simultaneamente uma multiplicidade de acções e que sejam envolvidos diversos intervenientes, as exigências que se imprimem à intervenção podem ser difíceis de operacionalizar e de implementar. Do nosso ponto de vista, parece colocar-se aos programas de intervenção o desafio de responder simultaneamente a duas exigências: serem suficientemente abrangentes, para abarcar as diferentes necessidades das crianças e das suas famílias, e serem suficientemente específicos, de forma a ir ao encontro das necessidades individuais de cada criança e da sua família. Como referem Sameroff e Fiese (2000), as intervenções não são extrinsecamente eficazes, é na sua interdependência com as particularidades dos participantes e de cada situação específica que podem surgir como tal.

Parece-nos que os programas de intervenção desenvolvidos a nível da comunidade poderão constituir uma boa resposta a esta dupla exigência. A comunidade é um campo de acção privilegiado por incorporar diversos serviços - escola, centro de saúde, etc. - ao mesmo tempo que corresponde a um contexto suficientemente próximo da vida das pessoas e no qual estas 
participam directamente (Garbarino \& Ganzel, 2000). Neste sentido, poder-se-ão implementar em cada comunidade múltiplas acções que funcionem de forma articulada e optimizada e que, ao mesmo tempo, sejam adequadas às especificidades das crianças e das famílias, como forma de potenciar o desenvolvimento de todas as crianças. Ao serem criados diferentes recursos nas comunidades, contribui-se, para além da prevenção de resultados negativos, para a criação de oportunidades (Dunst, Trivette, \& Deal, 1994).

De entre os vários serviços disponíveis na comunidade que, desenvolvendo a sua acção em articulação com outros, poderão assumir um papel preponderante, a escola merece uma especial atenção da nossa parte.

Tendo em conta que os resultados do presente estudo indicam que as competências mais baixas se relacionam com factores relativos ao contexto familiar das crianças, poder-se-á questionar o poder da escola para inverter a propensão de algumas crianças para o insucesso escolar. Poderá a escola, com o papel que lhe é historicamente atribuído - ensinar - exercer influência suficiente no desenvolvimento das crianças, a ponto de alterar este rumo? Parece-nos que o poder de acção de uma escola estará comprometido se não existirem simultaneamente outras acções de apoio e de intervenção junto das famílias e da comunidade em que estão inseridas. Acreditamos pois que, para responder eficazmente a estes problemas, a escola terá que se dotar dos meios necessários (nomeadamente, de meios técnicos e de equipas interdisciplinares), de modo a oferecer um serviço que promova o desenvolvimento de todas as crianças, o que implica, dado o desenvolvimento ser interdependente do contexto familiar e cultural, desenvolver acções direccionadas para as famílias e para as comunidades educativas. Não podendo responder a todos os problemas familiares, a escola poderá, no entanto, ao fomentar diferentes parcerias com a comunidade, facilitar a articulação entre os recursos existentes e as necessidades de cada família.

Apesar de se poderem concentrar na escola diferentes recursos e respostas às necessidades das crianças, consideramos, contudo, que a realidade é complexa e só com acções articuladas entre os mais diversos intervenientes, desde as mais distais - como decisões políticas - às mais próximas da criança - opções familiares - se consegue definitivamente alterar o rumo de desenvolvimento e impedir que existam crianças com insucesso educativo e que possam ver, assim, o seu futuro comprometido.

Por último, consideramos importante mencionar que o presente estudo não englobou algumas variáveis identificadas pela literatura como influentes no desenvolvimento da criança (Garbarino \& Ganzel, 2000; Sameroff \& Fiese, 2000), nomeadamente os processos interactivos que ocorrem entre a criança e os pais, ou o ambiente e a cultura da comunidade, aspectos que podem mediar e serem responsáveis por uma maior ou menor capacidade das famílias em dar resposta às necessidades desenvolvimentais da criança (Gar- 
barino \& Ganzel, 2000). Também não foi possível, no âmbito deste estudo, analisar os efeitos de alguns factores protectores de desenvolvimento, nomeadamente, o número de anos a frequentar o jardim-de-infância e que poderão alterar o impacto negativo dos factores de risco (Dunst et al., 1994;). Consideramos ser necessário desenvolver mais estudos que incorporem um maior número de variáveis e que analisem simultaneamente os factores de risco, promotores e protectores dos contextos principais de vida, para ganhar uma visão mais ampla das inter-relações entre esses diferentes factores. Apesar destas limitações, o nosso estudo contribui para confirmar o impacto negativo da acumulação de factores de risco no desenvolvimento das crianças e para salientar a necessidade de prevenir e de intervir de forma multifacetada e o mais precocemente possível.

\section{Referências}

Abreu-Lima, I., Oliveira, C., \& Tormenta, N. (2006). Avaliação de competências de numeracia. Poster apresentado na XI Conferência Internacional de Avaliação Psicológica: Formas e Contextos, Braga.

Abreu-Lima, I., \& Cadima, J. (2006). Comportamentos das crianças na sala de jardim-de-infância: A importância das competências sociais. In N. R. Santos, M. L. Lima, M. M. Melo, A. A. Candeias, M. L. Grácio, \& A. A. Calada (Orgs.), Actas do VI Simpósio Nacional de Investigação Em Psicologia. Évora: Departamento de Psicologia, Universidade de Évora (CD-ROM).

Ackerman, B. P., Izard, C. E., Schoff, K., Youngstrom, E. A., \& Kogos, J. (1999). Contextual Risk, and the problem behaviours of six- and seven-year-old children from economically disadvantaged families. Child Development, 70, $1415-$ $-1427$.

Burchinal, M. R., Roberts, J. E., Hooper, S., \& Zeisel, S. A. (2000). Cumulative risk and early cognitive development: A comparison of statistical risk models. Developmental Psychology, 36, 793-807.

Cadima, J., Gamelas, A. M., Leal, T., Silva, M., \& Peixoto, C. (2006). Desenvolvimento da Literacia Emergente: Qualidade dos contextos educativos e competências das crianças em idade pré-escolar. In N. R. Santos, M. L. Lima, M. M. Melo, A. A. Candeias, M. L. Grácio \& A. A. Calada (Orgs.), Actas do VI Simpósio Nacional de Investigação Em Psicologia. Évora: Departamento de Psicologia, Universidade de Évora (CD-ROM).

Castro, S. A., Cary, L., \& Gomes, I. (1998). Provas de Avaliação da Leitura - Nível Principiante. (Versão para Investigação. Porto: FPCEUP, Laboratório da Fala).

Clay, M. M. (2000). Segue-me, Lua: Adaptação portuguesa (Follow me moon; R. Alves \& C. Aguiar, Trans.). Porto, Portugal: Universidade do Porto, Centro de Psicologia.

Cohen, J. (1992). Quantitative methods in psychology: A power primer. Psychological Bulletin, 112, 155-159. 
Dunn, L. M. (1986). Test de vocabulario en imagénes Peabody. Adaptación española. Madrid: MEPSA (Traducción de Santiago Pereda).

Dunst, C. J., Trivette, C. M., \& Deal, A. G. (1994). Supporting \& Strengthening Families: Methods, strategies and practices. Cambridge: Brookline Books.

ECCE-Study Group (1997). European Child Care and Education: Cross national analyses of the quality and effects of different types early childhood programs on children's development (Final report package). European Union DG XII: Science, Research and Development. RTD Action: Targeted Socio-Economic Research.

Garbarino, J., \& Gazel, B. (2000). The human ecology of early risk. In J. P. Shonkoff \& S. J. Meisels (Eds), Handbook of early childhood intervention ( $2^{\text {nd }}$ Ed., pp. 76-93). Cambridge: Cambridge University Press.

Gutman, L. M., Sameroff, A. J., \& Cole, R. (2003). Academic growth curve trajectories from $1^{\text {st }}$ grade to $12^{\text {th }}$ grade: Effects of multiple social risk factors and preschool child factors. Developmental Psychology, 39, 777-790.

Gutman, L. M., Sameroff, A. J., \& Eccles, J. S. (2002). The academic achievement of African American students during early adolescence: An examination of multiple risk, promotive, and protective factors. American Journal of Community Psychology, 30, 367-399.

Hooper, S. R., Burchinal, M. R., Roberts, J. E., Zeisel, S., \& Neebe, E. C. (1998). Social and family risk factors for infant development at one year: An application of the cumulative risk model. Journal of Applied Developmental Psychology, 19, 85-96.

Lerner, R. M. (1997). Problems and potentials of youth development: A developmental contextual model for research and outreach promoting positive youth development. In J. L. Paul, M. Churton, H. Rosseli-Kosraryz, W. Marse et al. (Eds.), Foundations of special education (pp. 157-179). Boston: Brooks.

Peixoto, C., Silva, M., Leal, T., \& Cadima, J. (2008). Desenvolvimento da literacia emergente: Competências em crianças de idade pré-escolar. Actas do $6^{\circ}$ Encontro Nacional $/ 4^{\circ}$ Internacional de Investigação em Leitura, Literatura Infantil \& Ilustração. Braga: Centro de Estudos da Criança (CD-ROM).

Pianta, R. C., \& Walsh, D. J. (1996). High-Risk Children in Schools: Constructing sustaining relationships. London: Routledge.

Sameroff, A. J., \& Fiese, B. H. (2000). Transactional regulation: The developmental ecology in early intervention. In J. P. Shonkoff \& S. J. Meisels (Eds), Handbook of early childhood intervention ( $2^{\text {nd }}$ Ed., pp. 135-159). Cambridge: Cambridge University Press.

Sameroff, A. J., Seifer, R., Baldwin, A., \& Baldwin, C. (1993). Stability of intelligence from preschool to adolescence: The influence of social and family risk factors. Child Development, 86, 80-97.

Schaefer, E., Edgerton, M., \& Aaronson, M. R. (1978). Classroom Behaviour Inventory. Unpublished Rating Scale.

Sucena, A., \& Castro, S. L. (2001). Provas de avaliação da consciência fonológica. (Versão para Investigação). Porto: FPCEUP, Laboratório da Fala. 